\title{
Generating Set of the Complete Semigroups of Binary Relations
}

\author{
Yasha Diasamidze1, Neset Aydin², Ali Erdoğan ${ }^{3}$ \\ ${ }^{1}$ Shota Rustavelli University, Batumi, Georgia \\ ${ }^{2}$ Çanakkale Onsekiz Mart University, Çanakkale, Turkey \\ ${ }^{3}$ Hacettepe University, Ankara, Turkey \\ Email: diasamidze_ya@mail.ru,neseta@comu.edu.tr, alier@hacettepe.edu.tr
}

Received 16 December 2015; accepted 25 January 2016; published 28 January 2016

Copyright (C) 2016 by authors and Scientific Research Publishing Inc.

This work is licensed under the Creative Commons Attribution International License (CC BY).

http://creativecommons.org/licenses/by/4.0/

(c) (i) Open Access

\section{Abstract}

Difficulties encountered in studying generators of semigroup $B_{X}(D)$ of binary relations defined by a complete $X$-semilattice of unions $D$ arise because of the fact that they are not regular as a rule, which makes their investigation problematic. In this work, for special $D$, it has been seen that the semigroup $B_{X}(D)$, which are defined by semilattice $D$, can be generated by the set

$B=\left\{\alpha \in B_{X}(D) \mid V\left(X^{*}, \alpha\right)=D\right\}$.

\section{Keywords}

Semigroups, Binary Relation, Generated Set, Generators

\section{Introduction}

Theorem 1. Let $D=\left\{\breve{D}, Z_{1}, Z_{2}, \cdots, Z_{m-1}\right\}$ be some finite $X$-semilattice of unions and

$$
C(D)=\left\{P_{0}, P_{1}, P_{2}, \cdots, P_{m-1}\right\}
$$

be the family of sets of pairwise nonintersecting subsets of the set $X$.

If $\varphi$ is a mapping of the semilattice $D$ on the family of sets $C(D)$ which satisfies the condition $\varphi(\breve{D})=P_{0}$ and $\varphi\left(Z_{i}\right)=P_{i}$ for any $i=1,2, \cdots, m-1$ and $\hat{D}_{z}=D \backslash D_{T}$, then the following equalities are valid:

$$
\begin{aligned}
& \breve{D}=P_{0} \cup P_{1} \cup P_{2} \cup \cdots \cup P_{m-1}, \\
& Z_{i}=P_{0} \cup \bigcup_{T \in \hat{D}_{Z_{i}}} \varphi(T) .
\end{aligned}
$$


In the sequel these equalities will be called formal.

It is proved that if the elements of the semilattice $D$ are represented in the form 1 , then among the parameters $P_{i}(i=0,1,2, \cdots, m-1)$ there exist such parameters that cannot be empty sets for $D$. Such sets $P_{i}(0<i \leq m-1)$ are called basis sources, whereas sets $P_{i}(0 \leq j \leq m-1)$ which can be empty sets too are called completeness sources.

It is proved that under the mapping $\varphi$ the number of covering elements of the pre-image of a basis source is always equal to one, while under the mapping $\varphi$ the number of covering elements of the pre-image of a completeness source either does not exist or is always greater than one (see [1], Chapter 11). Some positive results in this direction can be found in [2]-[6].

Let $P_{0}, P_{1}, P_{2}, \cdots, P_{m-1}$ be parameters in the formal equalities, $\beta \in B_{X}(D)$ and

$$
\begin{aligned}
& \bar{\beta}=\bigcup_{i=0}^{m-1}\left(P_{i} \times \bigcup_{t \in P_{i}} t \beta\right) \cup \bigcup_{t^{\prime} \in X \backslash \bar{D}}\left(\left\{t^{\prime}\right\} \times t^{\prime} \beta\right) . \\
& \tilde{\beta}=\bigcup_{i=0}^{m-1}\left(P_{i} \times \bigcup_{t \in P_{i}} t \beta\right) \cup((X \backslash \breve{D}) \times \breve{D})
\end{aligned}
$$

The representation of the binary relation $\beta$ of the form $\bar{\beta}$ and $\tilde{\beta}$ will be called subquasinormal and maximal subquasinormal.

If $\bar{\beta}$ and $\tilde{\beta}$ are the subquasinormal and maximal subquasinormal representations of the binary relation $\beta$, then for the binary relations $\bar{\beta}$ and $\tilde{\beta}$ the following statements are true:

a) $\bar{\beta}, \tilde{\beta} \in B_{X}(D)$;

b) $\beta \subseteq \bar{\beta} \subseteq \tilde{\beta}$;

c) the subquasinormal representation of the binary relation $\beta$ is quasinormal;

d) if

$$
\bar{\beta}_{1}=\left(\begin{array}{cccc}
P_{0} & P_{1} & \cdots & P_{m-1} \\
P_{0} \bar{\beta} & P_{1} \bar{\beta} & \cdots & P_{m-1} \bar{\beta}
\end{array}\right),
$$

then $\bar{\beta}_{1}$ is a mapping of the family of sets $C(D)=\left\{P_{0}, P_{1}, P_{2}, \cdots, P_{m-1}\right\}$ in the $X$-semilattice of unions

$$
D=\left\{\breve{D}, Z_{1}, Z_{2}, \cdots, Z_{m-1}\right\} .
$$

e) if $\bar{\beta}_{2}: X \backslash \breve{D} \rightarrow D$ is a mapping satisfying the condition $\bar{\beta}_{2}\left(t^{\prime}\right)=t^{\prime} \beta$ for all $t^{\prime} \in X \backslash \breve{D}$, then

$$
\bar{\beta}=\bigcup_{i=0}^{m-1}\left(P_{i} \times \bigcup_{t \in P_{i}} t \beta\right) \cup \bigcup_{t^{\prime} \in X \backslash \bar{D}}\left(\left\{t^{\prime}\right\} \times \bar{\beta}_{2}\left(t^{\prime}\right)\right) .
$$

\section{Results}

Proposition 2. Let $\alpha, \beta \in B_{X}(D)$. Then

$$
\alpha \circ \beta=\alpha \circ \bar{\beta}=\alpha \circ \tilde{\beta}
$$

Proof. It is easy to see the inclusion $\alpha \circ \beta \subseteq \alpha \circ \bar{\beta} \subseteq \alpha \circ \tilde{\beta}$ holds, since $\beta \subseteq \bar{\beta} \subseteq \tilde{\beta}$. If $x(\alpha \circ \tilde{\beta}) y$ $(x, y \in X)$, then $x \alpha z \tilde{\beta} y$ for some $z \in X$. So, $z \notin X \backslash \breve{D}$ since $z \in x \alpha \in D$ and $z\left(\bigcup_{i=0}^{m-1}\left(P_{i} \times \bigcup_{t \in P_{i}} t \beta\right)\right) y$.

Then $z\left(P_{k} \times \bigcup_{t \in P_{k}} t \beta\right) y$ for some $k(0 \leq k \leq m-1)$ i.e. $z \in P_{k}$ and $y \in z \beta \subseteq \bigcup_{t \in P_{k}} t \beta$. For the last condition follows that $z \beta y$. We have $x \alpha z \beta y$ and $x(\alpha \circ \beta) y$. Therefore, the inclusion $\alpha \circ \tilde{\beta} \subseteq \alpha \circ \beta$ is true. Of this and by inclusion $\alpha \circ \beta \subseteq \alpha \circ \bar{\beta} \subseteq \alpha \circ \tilde{\beta}$ follows that the equality $\alpha \circ \beta=\alpha \circ \bar{\beta}=\alpha \circ \tilde{\beta}$ holds.

Corollary 1. If $\alpha, \delta, \beta \in B_{X}(D)$ and $\beta \subseteq \delta \subseteq \tilde{\beta}$, then $\alpha \circ \beta=\alpha \circ \delta=\alpha \circ \tilde{\beta}$.

Proof. We have $\beta \subseteq \delta \subseteq \tilde{\beta}$ and $\alpha \circ \beta \subseteq \alpha \circ \delta \subseteq \alpha \circ \tilde{\beta}$. Of this follows that $\alpha \circ \beta=\alpha \circ \delta=\alpha \circ \tilde{\beta}$ since $\alpha \circ \beta=\alpha \circ \tilde{\beta}$.

Let the $X$-semilattice $D=\left\{Z_{4}, Z_{3}, Z_{2}, Z_{1}, \breve{D}\right\}$ of unions given by the diagram of Figure 1. Formal equalities of the given semilattice have a form: 


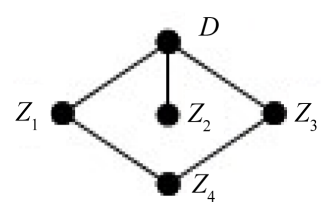

Figure 1. Diagram of D.

$$
\begin{aligned}
& \breve{D}=P_{0} \cup P_{1} \cup P_{2} \cup P_{3} \cup P_{4}, \\
& Z_{1}=P_{0} \cup P_{2} \cup P_{3} \cup P_{4}, \\
& Z_{2}=P_{0} \cup P_{1} \cup P_{3} \cup P_{4}, \\
& Z_{3}=P_{0} \cup P_{1} \cup P_{2} \cup P_{4}, \\
& Z_{4}=P_{0} \cup P_{2} .
\end{aligned}
$$

The parameters $P_{1}, P_{2}, P_{3}$ are basis sources and the parameters $P_{0}, P_{4}$ are completeness sources, i.e. $|X| \geq 3$.

Example 3. Let $X=\{1,2,3,4,5,6,7\}, P_{1}=\{1,4\}, P_{2}=\{2,5\}, P_{3}=\{3,6\}, P_{0}=P_{4}=\varnothing$. Then for the formal equalities of the semilattice $D$ follows that $Z_{4}=\{2,5\}, Z_{3}=\{1,2,4,5\}, Z_{2}=\{1,3,4,6\}, Z_{1}=\{2,3,5,6\}$, $\breve{D}=\{1,2,3,4,5,6\}, \quad D=\{\{2\},\{1,2\},\{1,3\},\{2,3\},\{1,2,3\}\}$, and $\beta=(\{1\} \times\{2,3,5,6\}) \cup(\{2\} \times\{1,3,4,6\}) \cup(\{3\} \times\{1,2,4,5\}) \cup(\{4,5,6,7\} \times\{2,5\})$.

Then we have:

$$
\begin{aligned}
& \bigcup_{t \in P_{1}} t \beta=\{2,3,5,6\}, \bigcup_{t \in P_{2}} t \beta=\{1,2,3,4,5,6\}, \bigcup_{t \in P_{3}} t \beta=\{1,2,4,5\}, \\
& \bigcup_{t \in P_{0}} t \beta=\bigcup_{t \in P_{4}} t \beta=\varnothing ; \\
& \bar{\beta}=(\{1,4\} \times\{2,3,5,6\}) \cup(\{2,5\} \times\{1,2,3,4,5,6\}) \cup(\{3,6\} \times\{1,2,4,5\}) \cup(\{7\} \times\{2,5\}) ; \\
& \tilde{\beta}=(\{1,4\} \times\{2,3,5,6\}) \cup(\{2,5\} \times\{1,2,3,4,5,6\}) \cup(\{3,6\} \times\{1,2,4,5\}) \cup(\{7\} \times\{1,2,3,4,5,6,7\}) .
\end{aligned}
$$

Theorem 4. Let the $X$-semilattice $D=\left\{Z_{4}, Z_{3}, Z_{2}, Z_{1}, \breve{D}\right\}$ of unions given by the diagram of Figure 1 , $B=\left\{\alpha \in B_{X}(D) \mid V\left(X^{*}, \alpha\right)=D\right\}$ and $|X \backslash \breve{D}| \geq 3$. Then the set $B$ is generating set of the semigroup $B_{X}(D)$.

Proof. It is easy to see that $|X| \geq 6$ since $P_{1}, P_{2}, P_{3} \notin\{\varnothing\}$ and $|X \backslash \breve{D}| \geq 3$. Now, let $\alpha$ be any binary relation of the semigroup $B_{\underline{X}}(D) ; \alpha_{1}, \alpha_{2}, \cdots, \alpha_{n} \in B, \quad \alpha=\alpha_{1} \circ \alpha_{2} \circ \cdots \circ \alpha_{n}$ and $\beta=\alpha_{2} \circ \alpha_{3} \circ \cdots \circ \alpha_{n}$. Then the equality $\alpha=\alpha_{1} \circ \beta=\alpha_{1} \circ \bar{\beta}$ ( $\bar{\beta}$ is subquasinormal representation of a binary relation $\beta$ ) is true. By assumption $\alpha_{1} \in B_{X}(D)$, i.e. the quasinormal representation of a binary relation $\alpha_{1}$ have a form

$$
\alpha_{1}=\left(Y_{4}^{\alpha_{1}} \times Z_{4}\right) \cup\left(Y_{3}^{\alpha_{1}} \times Z_{3}\right) \cup\left(Y_{2}^{\alpha_{1}} \times Z_{2}\right) \cup\left(Y_{1}^{\alpha_{1}} \times Z_{1}\right) \cup\left(Y_{0}^{\alpha_{1}} \times \breve{D}\right) .
$$

Of this follows that

$$
\begin{aligned}
\alpha & =\alpha_{1} \circ \bar{\beta}=\left(Y_{4}^{\alpha_{1}} \times Z_{4}\right) \cup\left(Y_{3}^{\alpha_{1}} \times Z_{3}\right) \cup\left(Y_{2}^{\alpha_{1}} \times Z_{2}\right) \cup\left(Y_{1}^{\alpha_{1}} \times Z_{1}\right) \cup\left(Y_{0}^{\alpha_{1}} \times \breve{D}\right) \circ \bar{\beta} \\
& =\left(Y_{4}^{\alpha_{1}} \times Z_{4} \bar{\beta}\right) \cup\left(Y_{3}^{\alpha_{1}} \times Z_{3} \bar{\beta}\right) \cup\left(Y_{2}^{\alpha_{1}} \times Z_{2} \bar{\beta}\right) \cup\left(Y_{1}^{\alpha_{1}} \times Z_{1} \bar{\beta}\right) \cup\left(Y_{0}^{\alpha_{1}} \times \bar{D} \bar{\beta}\right) .
\end{aligned}
$$

For the binary relation $\alpha$ we consider the following case.

a) Let $\left|V\left(X^{*}, \alpha\right)\right|=1$. Then $\alpha=X \times T$, where $T \in D$. By element $T$ we consider the following cases:

1. $T \neq \breve{D}$. In this case suppose that

$$
\bar{\beta}_{1}=\left(\begin{array}{ccccc}
P_{0} & P_{1} & P_{2} & P_{3} & P_{4} \\
\varnothing & T & T & T & \varnothing
\end{array}\right)
$$

and $\bar{\beta}_{2}$ are mapping of the set $X \backslash \breve{D}$ on the set $\left\{Z_{4}, Z_{3}, Z_{2}, Z_{1}\right\} \backslash\{T\}$. Then 


$$
\begin{aligned}
& \bar{\beta}=\left(P_{0} \times \varnothing\right) \cup\left(P_{1} \times T\right) \cup\left(P_{2} \times T\right) \cup\left(P_{3} \times T\right) \cup\left(P_{4} \times \varnothing\right) \cup \bigcup_{t^{\prime} \in X \backslash \breve{D}}\left(\left\{t^{\prime}\right\} \times \bar{\beta}_{2}\left(t^{\prime}\right)\right), \\
& \alpha_{1}=\left(Y_{4}^{\alpha_{1}} \times Z_{4}\right) \cup\left(Y_{3}^{\alpha_{1}} \times Z_{3}\right) \cup\left(Y_{2}^{\alpha_{1}} \times Z_{2}\right) \cup\left(Y_{1}^{\alpha_{1}} \times Z_{1}\right) \cup\left(Y_{0}^{\alpha_{1}} \times \breve{D}\right),
\end{aligned}
$$

where $Y_{4}^{\alpha_{1}}, Y_{3}^{\alpha_{1}}, Y_{2}^{\alpha_{1}}, Y_{1}^{\alpha_{1}} \notin\{\varnothing\}$, then it is easy to see, that $\alpha_{1}, \bar{\beta} \in B$ since $V\left(D, \alpha_{1}\right)=V(D, \bar{\beta})=D$. From the formal equality and equalities (6) and (5) we have:

$$
\begin{aligned}
& Z_{4} \bar{\beta}=\left(P_{0} \cup P_{2}\right) \bar{\beta}=P_{0} \bar{\beta} \cup P_{2} \bar{\beta}=\varnothing \cup T=T ; \\
& Z_{3} \bar{\beta}=\left(P_{0} \cup P_{1} \cup P_{2} \cup P_{4}\right) \bar{\beta}=P_{0} \bar{\beta} \cup P_{1} \bar{\beta} \cup P_{2} \bar{\beta} \cup P_{4} \bar{\beta}=\varnothing \cup T \cup T \cup \varnothing=T ; \\
& Z_{2} \bar{\beta}=\left(P_{0} \cup P_{1} \cup P_{3} \cup P_{4}\right) \bar{\beta}=P_{0} \bar{\beta} \cup P_{1} \bar{\beta} \cup P_{3} \bar{\beta} \cup P_{4} \bar{\beta}=\varnothing \cup T \cup T \cup \varnothing=T ; \\
& Z_{1} \bar{\beta}=\left(P_{0} \cup P_{2} \cup P_{3} \cup P_{4}\right) \bar{\beta}=P_{0} \bar{\beta} \cup P_{2} \bar{\beta} \cup P_{3} \bar{\beta} \cup P_{4} \bar{\beta}=\varnothing \cup T \cup T \cup \varnothing=T ; \\
& \bar{D} \bar{\beta}=\left(P_{0} \cup P_{1} \cup P_{2} \cup P_{3} \cup P_{4}\right) \bar{\beta}=P_{0} \bar{\beta} \cup P_{1} \bar{\beta} \cup P_{2} \bar{\beta} \cup P_{3} \bar{\beta} \cup P_{4} \bar{\beta} \\
& \quad=\varnothing \cup T \cup T \cup T \cup \varnothing=T ; \\
& \alpha=\alpha_{1} \circ \bar{\beta} \\
& =\left(Y_{4}^{\alpha_{1}} \times Z_{4} \bar{\beta}\right) \cup\left(Y_{3}^{\alpha_{1}} \times Z_{3} \bar{\beta}\right) \cup\left(Y_{2}^{\alpha_{1}} \times Z_{2} \bar{\beta}\right) \cup\left(Y_{1}^{\alpha_{1}} \times Z_{1} \bar{\beta}\right) \cup\left(Y_{0}^{\alpha_{1}} \times \bar{D} \bar{\beta}\right) \\
& =\left(Y_{4}^{\alpha_{1}} \times T\right) \cup\left(Y_{3}^{\alpha_{1}} \times T\right) \cup\left(Y_{2}^{\alpha_{1}} \times T\right) \cup\left(Y_{1}^{\alpha_{1}} \times T\right) \cup\left(Y_{0}^{\alpha_{1}} \times T\right)=X \times T
\end{aligned}
$$

since $Y_{4}^{\alpha_{1}} \cup Y_{3}^{\alpha_{1}} \cup Y_{2}^{\alpha_{1}} \cup Y_{1}^{\alpha_{1}} \cup Y_{0}^{\alpha_{1}}=X$.

2. $T=\breve{D}$. In this case suppose that

$$
\bar{\beta}_{1}=\left(\begin{array}{lllll}
P_{0} & P_{1} & P_{2} & P_{3} & P_{4} \\
\breve{D} & Z_{1} & Z_{2} & Z_{3} & Z_{4}
\end{array}\right)
$$

and $\bar{\beta}_{2}$ are mapping of the set $X \backslash \breve{D}$ in the set $D$. Then

$$
\begin{aligned}
& \bar{\beta}=\left(P_{0} \times \breve{D}\right) \cup\left(P_{1} \times Z_{1}\right) \cup\left(P_{2} \times Z_{2}\right) \cup\left(P_{3} \times Z_{3}\right) \cup\left(P_{4} \times Z_{4}\right) \cup \bigcup_{t^{\prime} \in X \backslash \breve{D}}\left(\left\{t^{\prime}\right\} \times \bar{\beta}_{2}\left(t^{\prime}\right)\right), \\
& \alpha_{1}=\left(Y_{4}^{\alpha_{1}} \times Z_{4}\right) \cup\left(Y_{3}^{\alpha_{1}} \times Z_{3}\right) \cup\left(Y_{2}^{\alpha_{1}} \times Z_{2}\right) \cup\left(Y_{1}^{\alpha_{1}} \times Z_{1}\right) \cup\left(Y_{0}^{\alpha_{1}} \times \breve{D}\right),
\end{aligned}
$$

where $Y_{4}^{\alpha_{1}}, Y_{3}^{\alpha_{1}}, Y_{2}^{\alpha_{1}}, Y_{1}^{\alpha_{1}} \notin\{\varnothing\}$, then it is easy to see, that $\alpha_{1}, \bar{\beta} \in B$ since $V\left(D, \alpha_{1}\right)=V(D, \bar{\beta})=D$. From the formal equality and equalities (7) and (5) we have:

$$
\begin{aligned}
& Z_{4} \bar{\beta}=\left(P_{0} \cup P_{2}\right) \bar{\beta}=P_{0} \bar{\beta} \cup P_{2} \bar{\beta}=\breve{D} ; \\
& Z_{3} \bar{\beta}=\left(P_{0} \cup P_{1} \cup P_{2} \cup P_{4}\right) \bar{\beta}=P_{0} \bar{\beta} \cup P_{1} \bar{\beta} \cup P_{2} \bar{\beta} \cup P_{4} \bar{\beta}=\breve{D} ; \\
& Z_{2} \bar{\beta}=\left(P_{0} \cup P_{1} \cup P_{3} \cup P_{4}\right) \bar{\beta}=P_{0} \bar{\beta} \cup P_{1} \bar{\beta} \cup P_{3} \bar{\beta} \cup P_{4} \bar{\beta}=\breve{D} ; \\
& Z_{1} \bar{\beta}=\left(P_{0} \cup P_{2} \cup P_{3} \cup P_{4}\right) \bar{\beta}=P_{0} \bar{\beta} \cup P_{2} \bar{\beta} \cup P_{3} \bar{\beta} \cup P_{4} \bar{\beta}=\breve{D} ; \\
& \breve{D} \bar{\beta}=\left(P_{0} \cup P_{1} \cup P_{2} \cup P_{3} \cup P_{4}\right) \bar{\beta}=P_{0} \bar{\beta} \cup P_{1} \bar{\beta} \cup P_{2} \bar{\beta} \cup P_{3} \bar{\beta} \cup P_{4} \bar{\beta}=\breve{D} ; \\
& \alpha=\alpha_{1} \circ \bar{\beta} \\
& =\left(Y_{4}^{\alpha_{1}} \times Z_{4} \bar{\beta}\right) \cup\left(Y_{3}^{\alpha_{1}} \times Z_{3} \bar{\beta}\right) \cup\left(Y_{2}^{\alpha_{1}} \times Z_{2} \bar{\beta}\right) \cup\left(Y_{1}^{\alpha_{1}} \times Z_{1} \bar{\beta}\right) \cup\left(Y_{0}^{\alpha_{1}} \times \breve{D} \bar{\beta}\right) \\
& =\left(Y_{4}^{\alpha_{1}} \times \breve{D}\right) \cup\left(Y_{3}^{\alpha_{1}} \times \breve{D}\right) \cup\left(Y_{2}^{\alpha_{1}} \times \breve{D}\right) \cup\left(Y_{1}^{\alpha_{1}} \times \breve{D}\right) \cup\left(Y_{0}^{\alpha_{1}} \times \breve{D}\right) \\
& =X \times \breve{D} .
\end{aligned}
$$

b) $\left|V\left(X^{*}, \alpha\right)\right|=2$. Then

$$
V\left(X^{*}, \alpha\right) \in\left\{\left\{Z_{4}, Z_{1}\right\},\left\{Z_{4}, Z_{3}\right\},\left\{Z_{4}, \breve{D}\right\},\left\{Z_{3}, \breve{D}\right\},\left\{Z_{2}, \breve{D}\right\},\left\{Z_{1}, \breve{D}\right\}\right\}
$$

since $V\left(X^{*}, \alpha\right)$ is $X$-semilattice of unions. For the semilattice of unions $V\left(X^{*}, \alpha\right)$ consider the following cases.

1. Let $V\left(X^{*}, \alpha\right)=\left\{Z_{4}, T\right\}$, where, $T \in\left\{Z_{1}, Z_{3}\right\}$. Then binary relation $\alpha$ has representation of the form 
$\alpha=\left(Y_{4}^{\alpha} \times Z_{4}\right) \cup\left(Y_{T}^{\alpha} \times T\right)$. In this case suppose that

$$
\bar{\beta}_{1}=\left(\begin{array}{ccccc}
P_{0} & P_{1} & P_{2} & P_{3} & P_{4} \\
\varnothing & T & Z_{4} & T & \varnothing
\end{array}\right)
$$

and $\bar{\beta}_{2}$ are mapping of the set $X \backslash \breve{D}$ on the set $D \backslash\left\{Z_{4}, T\right\}$. Then

$$
\begin{aligned}
& \bar{\beta}=\left(P_{0} \times \varnothing\right) \cup\left(P_{1} \times T\right) \cup\left(P_{2} \times Z_{4}\right) \cup\left(P_{3} \times T\right) \cup\left(P_{4} \times \varnothing\right) \cup \bigcup_{t^{\prime} \in X \backslash \bar{D}}\left(\left\{t^{\prime}\right\} \times \bar{\beta}_{2}\left(t^{\prime}\right)\right), \\
& \alpha_{1}=\left(Y_{4}^{\alpha_{1}} \times Z_{4}\right) \cup\left(Y_{3}^{\alpha_{1}} \times Z_{3}\right) \cup\left(Y_{2}^{\alpha_{1}} \times Z_{2}\right) \cup\left(Y_{1}^{\alpha_{1}} \times Z_{1}\right) \cup\left(Y_{0}^{\alpha_{1}} \times \breve{D}\right),
\end{aligned}
$$

where $Y_{4}^{\alpha_{1}}, Y_{3}^{\alpha_{1}}, Y_{2}^{\alpha_{1}}, Y_{1}^{\alpha_{1}} \notin\{\varnothing\}, Y_{4}^{\alpha_{1}}=Y_{4}^{\alpha}$ and $Y_{3}^{\alpha_{1}} \cup Y_{2}^{\alpha_{1}} \cup Y_{1}^{\alpha_{1}} \cup Y_{0}^{\alpha_{1}}=Y_{0}^{\alpha}$, then it is easy to see, that $\alpha_{1}, \bar{\beta} \in B$ since $V\left(D, \alpha_{1}\right)=V(D, \bar{\beta})=D$. From the formal equality and equalities (8) and (5) we have:

$$
\begin{aligned}
Z_{4} \bar{\beta} & =\left(P_{0} \cup P_{2}\right) \bar{\beta}=P_{0} \bar{\beta} \cup P_{2} \bar{\beta}=\varnothing \cup Z_{4}=Z_{4} ; \\
Z_{3} \bar{\beta} & =\left(P_{0} \cup P_{1} \cup P_{2} \cup P_{4}\right) \bar{\beta}=P_{0} \bar{\beta} \cup P_{1} \bar{\beta} \cup P_{2} \bar{\beta} \cup P_{4} \bar{\beta} \\
& =\varnothing \cup T \cup Z_{4} \cup \varnothing=T ; \\
Z_{2} \bar{\beta} & =\left(P_{0} \cup P_{1} \cup P_{3} \cup P_{4}\right) \bar{\beta}=P_{0} \bar{\beta} \cup P_{1} \bar{\beta} \cup P_{3} \bar{\beta} \cup P_{4} \bar{\beta} \\
& =\varnothing \cup T \cup T \cup \varnothing=T ; \\
Z_{1} \bar{\beta} & =\left(P_{0} \cup P_{2} \cup P_{3} \cup P_{4}\right) \bar{\beta}=P_{0} \bar{\beta} \cup P_{2} \bar{\beta} \cup P_{3} \bar{\beta} \cup P_{4} \bar{\beta} \\
& =\varnothing \cup Z_{4} \cup T \cup \varnothing=T ; \\
\bar{D} \bar{\beta} & =\left(P_{0} \cup P_{1} \cup P_{2} \cup P_{3} \cup P_{4}\right) \bar{\beta}=P_{0} \bar{\beta} \cup P_{1} \bar{\beta} \cup P_{2} \bar{\beta} \cup P_{3} \bar{\beta} \cup P_{4} \bar{\beta} \\
& =\varnothing \cup T \cup Z_{4} \cup T \cup \varnothing=T ; \\
\alpha & =\alpha_{1} \circ \bar{\beta} \\
& =\left(Y_{4}^{\alpha_{1}} \times Z_{4} \bar{\beta}\right) \cup\left(Y_{3}^{\alpha_{1}} \times Z_{3} \bar{\beta}\right) \cup\left(Y_{2}^{\alpha_{1}} \times Z_{2} \bar{\beta}\right) \cup\left(Y_{1}^{\alpha_{1}} \times Z_{1} \bar{\beta}\right) \cup\left(Y_{0}^{\alpha_{1}} \times \bar{D} \bar{\beta}\right) \\
& =\left(Y_{4}^{\alpha_{1}} \times Z_{4}\right) \cup\left(Y_{3}^{\alpha_{1}} \times T\right) \cup\left(Y_{2}^{\alpha_{1}} \times T\right) \cup\left(Y_{1}^{\alpha_{1}} \times T\right) \cup\left(Y_{0}^{\alpha_{1}} \times T\right) \\
& =\left(Y_{4}^{\alpha_{1}} \times Z_{4}\right) \cup\left(\left(Y_{3}^{\alpha_{1}} \cup Y_{2}^{\alpha_{1}} \cup Y_{1}^{\alpha_{1}} \cup Y_{0}^{\alpha_{1}}\right) \times T\right)=\left(Y_{4}^{\alpha} \times Z_{4}\right) \cup\left(Y_{0}^{\alpha} \times T\right) .
\end{aligned}
$$

2. Let $V\left(X^{*}, \alpha\right)=\{T, \breve{D}\}$, where, $T \in\left\{Z_{4}, Z_{3}, Z_{2}, Z_{1}\right\}$. Then binary relation $\alpha$ has representation of the form $\alpha=\left(Y_{T}^{\alpha} \times T\right) \cup\left(Y_{0}^{\alpha} \times \breve{D}\right)$. In this case suppose that

$$
\bar{\beta}_{1}=\left(\begin{array}{ccccc}
P_{0} & P_{1} & P_{2} & P_{3} & P_{4} \\
\varnothing & \breve{D} & T & \breve{D} & \varnothing
\end{array}\right)
$$

and $\bar{\beta}_{2}$ are mapping of the set $X \backslash \breve{D}$ on the set $D \backslash\{T, \breve{D}\}$. Then

$$
\begin{aligned}
& \bar{\beta}=\left(P_{0} \times \varnothing\right) \cup\left(P_{1} \times \breve{D}\right) \cup\left(P_{2} \times T\right) \cup\left(P_{3} \times \breve{D}\right) \cup\left(P_{4} \times \varnothing\right) \cup \bigcup_{t^{\prime} \in X \backslash \breve{D}}\left(\left\{t^{\prime}\right\} \times f\left(t^{\prime}\right)\right), \\
& \alpha_{1}=\left(Y_{4}^{\alpha_{1}} \times Z_{4}\right) \cup\left(Y_{3}^{\alpha_{1}} \times Z_{3}\right) \cup\left(Y_{2}^{\alpha_{1}} \times Z_{2}\right) \cup\left(Y_{1}^{\alpha_{1}} \times Z_{1}\right) \cup\left(Y_{0}^{\alpha_{1}} \times \breve{D}\right),
\end{aligned}
$$

where $Y_{4}^{\alpha_{1}}, Y_{3}^{\alpha_{1}}, Y_{2}^{\alpha_{1}}, Y_{1}^{\alpha_{1}} \notin\{\varnothing\}, Y_{4}^{\alpha_{1}}=Y_{T}^{\alpha}$ and $Y_{3}^{\alpha_{1}} \cup Y_{2}^{\alpha_{1}} \cup Y_{1}^{\alpha_{1}} \cup Y_{0}^{\alpha_{1}}=Y_{0}^{\alpha}$, then it is easy to see, that $\alpha_{1}, \bar{\beta} \in B$ since $V\left(D, \alpha_{1}\right)=V(D, \bar{\beta})=D$. From the formal equality and equalities (9) and (5) we have: 


$$
\begin{aligned}
& Z_{4} \bar{\beta}=\left(P_{0} \cup P_{2}\right) \bar{\beta}=P_{0} \bar{\beta} \cup P_{2} \bar{\beta}=\varnothing \cup T=T ; \\
& Z_{3} \bar{\beta}=\left(P_{0} \cup P_{1} \cup P_{2} \cup P_{4}\right) \bar{\beta}=P_{0} \bar{\beta} \cup P_{1} \bar{\beta} \cup P_{2} \bar{\beta} \cup P_{4} \bar{\beta}=\varnothing \cup \breve{D} \cup T \cup \varnothing=\breve{D} ; \\
& Z_{2} \bar{\beta}=\left(P_{0} \cup P_{1} \cup P_{3} \cup P_{4}\right) \bar{\beta}=P_{0} \bar{\beta} \cup P_{1} \bar{\beta} \cup P_{3} \bar{\beta} \cup P_{4} \bar{\beta}=\varnothing \cup \breve{D} \cup \breve{D} \cup \varnothing=\breve{D} ; \\
& Z_{1} \bar{\beta}=\left(P_{0} \cup P_{2} \cup P_{3} \cup P_{4}\right) \bar{\beta}=P_{0} \bar{\beta} \cup P_{2} \bar{\beta} \cup P_{3} \bar{\beta} \cup P_{4} \bar{\beta}=\varnothing \cup T \cup \breve{D} \cup \varnothing=\breve{D} ; \\
& \bar{D} \bar{\beta}=\left(P_{0} \cup P_{1} \cup P_{2} \cup P_{3} \cup P_{4}\right) \bar{\beta}=P_{0} \bar{\beta} \cup P_{1} \bar{\beta} \cup P_{2} \bar{\beta} \cup P_{3} \bar{\beta} \cup P_{4} \bar{\beta} \\
&=\varnothing \cup \breve{D} \cup T \cup \breve{D} \cup \varnothing=\breve{D} ; \\
& \alpha= \alpha_{1} \circ \bar{\beta} \\
&=\left(Y_{4}^{\alpha_{1}} \times Z_{4} \bar{\beta}\right) \cup\left(Y_{3}^{\alpha_{1}} \times Z_{3} \bar{\beta}\right) \cup\left(Y_{2}^{\alpha_{1}} \times Z_{2} \bar{\beta}\right) \cup\left(Y_{1}^{\alpha_{1}} \times Z_{1} \bar{\beta}\right) \cup\left(Y_{0}^{\alpha_{1}} \times \breve{D} \bar{\beta}\right) \\
&=\left(Y_{4}^{\alpha_{1}} \times T\right) \cup\left(Y_{3}^{\alpha_{1}} \times \breve{D}\right) \cup\left(Y_{2}^{\alpha_{1}} \times \breve{D}\right) \cup\left(Y_{1}^{\alpha_{1}} \times \breve{D}\right) \cup\left(Y_{0}^{\alpha_{1}} \times \breve{D}\right) \\
&=\left(Y_{4}^{\alpha_{1}} \times T\right) \cup\left(\left(Y_{3}^{\alpha_{1}} \cup Y_{2}^{\alpha_{1}} \cup Y_{1}^{\alpha_{1}} \cup Y_{0}^{\alpha_{1}}\right) \times \breve{D}\right)=\left(Y_{T}^{\alpha} \times T\right) \cup\left(Y_{0}^{\alpha} \times \breve{D}\right) . \\
&\left.x^{2}\right) \mid=3 . \text { Then } \\
& V\left(X^{*}, \alpha\right) \in\left\{\left\{Z_{4}, Z_{1}, \breve{D}\right\},\left\{Z_{4}, Z_{3}, \breve{D}\right\},\left\{Z_{4}, Z_{2}, \breve{D}\right\},\left\{Z_{1}, Z_{2}, \breve{D}\right\},\left\{Z_{1}, Z_{3}, \breve{D}\right\},\left\{Z_{2}, Z_{3}, \breve{D}\right\}\right\}
\end{aligned}
$$

c) $\left|V\left(X^{*}, \alpha\right)\right|=3$. Then

since $V\left(X^{*}, \alpha\right)$ is $X$-semilattice of unions. For the semilattice of unions $V\left(X^{*}, \alpha\right)$ consider the following cases.

1. Let $V\left(X^{*}, \alpha\right)=\left\{Z_{4}, Z_{1}, \breve{D}\right\}$. Then binary relation $\alpha$ has representation of the form $\alpha=\left(Y_{4}^{\alpha} \times Z_{4}\right) \cup\left(Y_{1}^{\alpha} \times Z_{1}\right) \cup\left(Y_{0}^{\alpha} \times \breve{D}\right)$. In this case suppose that

$$
\bar{\beta}_{1}=\left(\begin{array}{lllll}
P_{0} & P_{1} & P_{2} & P_{3} & P_{4} \\
\varnothing & Z_{2} & Z_{4} & Z_{1} & \varnothing
\end{array}\right)
$$

and $\bar{\beta}_{2}$ are mapping of the set $X \backslash \breve{D}$ on the set $D \backslash\left\{Z_{4}, Z_{2}, Z_{1}\right\}$. Then

$$
\begin{aligned}
& \bar{\beta}=\left(P_{0} \times \varnothing\right) \cup\left(P_{1} \times Z_{2}\right) \cup\left(P_{2} \times Z_{4}\right) \cup\left(P_{3} \times Z_{1}\right) \cup\left(P_{4} \times \varnothing\right) \cup \bigcup_{t^{\prime} \in X \backslash \bar{D}}\left(\left\{t^{\prime}\right\} \times f\left(t^{\prime}\right)\right), \\
& \alpha_{1}=\left(Y_{4}^{\alpha_{1}} \times Z_{4}\right) \cup\left(Y_{3}^{\alpha_{1}} \times Z_{3}\right) \cup\left(Y_{2}^{\alpha_{1}} \times Z_{2}\right) \cup\left(Y_{1}^{\alpha_{1}} \times Z_{1}\right) \cup\left(Y_{0}^{\alpha_{1}} \times \breve{D}\right),
\end{aligned}
$$

where $Y_{4}^{\alpha_{1}}, Y_{3}^{\alpha_{1}}, Y_{2}^{\alpha_{1}}, Y_{1}^{\alpha_{1}} \notin\{\varnothing\}, Y_{4}^{\alpha_{1}}=Y_{4}^{\alpha}, Y_{1}^{\alpha_{1}}=Y_{1}^{\alpha}$ and $Y_{3}^{\alpha_{1}} \cup Y_{2}^{\alpha_{1}} \cup Y_{0}^{\alpha_{1}}=Y_{0}^{\alpha}$, then it is easy to see, that $\alpha_{1}, \bar{\beta} \in B$ since $V\left(D, \alpha_{1}\right)=V(D, \bar{\beta})=D$. From the formal equality and equalities (10) and (5) we have:

$$
\begin{aligned}
& Z_{4} \bar{\beta}=\left(P_{0} \cup P_{2}\right) \bar{\beta}=P_{0} \bar{\beta} \cup P_{2} \bar{\beta}=\varnothing \cup Z_{4}=Z_{4} ; \\
& Z_{3} \bar{\beta}=\left(P_{0} \cup P_{1} \cup P_{2} \cup P_{4}\right) \bar{\beta}=P_{0} \bar{\beta} \cup P_{1} \bar{\beta} \cup P_{2} \bar{\beta} \cup P_{4} \bar{\beta}=\varnothing \cup Z_{2} \cup Z_{4} \cup \varnothing=\breve{D} ; \\
& Z_{2} \bar{\beta}=\left(P_{0} \cup P_{1} \cup P_{3} \cup P_{4}\right) \bar{\beta}=P_{0} \bar{\beta} \cup P_{1} \bar{\beta} \cup P_{3} \bar{\beta} \cup P_{4} \bar{\beta}=\varnothing \cup Z_{2} \cup Z_{1} \cup \varnothing=\breve{D} ; \\
& Z_{1} \bar{\beta}=\left(P_{0} \cup P_{2} \cup P_{3} \cup P_{4}\right) \bar{\beta}=P_{0} \bar{\beta} \cup P_{2} \bar{\beta} \cup P_{3} \bar{\beta} \cup P_{4} \bar{\beta}=\varnothing \cup Z_{4} \cup Z_{1} \cup \varnothing=Z_{1} ; \\
& \bar{D} \bar{\beta}=\left(P_{0} \cup P_{1} \cup P_{2} \cup P_{3} \cup P_{4}\right) \bar{\beta}=P_{0} \bar{\beta} \cup P_{1} \bar{\beta} \cup P_{2} \bar{\beta} \cup P_{3} \bar{\beta} \cup P_{4} \bar{\beta} \\
& \quad=\varnothing \cup Z_{2} \cup Z_{4} \cup Z_{1} \cup \varnothing=\bar{D} ; \\
& \alpha=\alpha_{1} \circ \bar{\beta} \\
& =\left(Y_{4}^{\alpha_{1}} \times Z_{4} \bar{\beta}\right) \cup\left(Y_{3}^{\alpha_{1}} \times Z_{3} \bar{\beta}\right) \cup\left(Y_{2}^{\alpha_{1}} \times Z_{2} \bar{\beta}\right) \cup\left(Y_{1}^{\alpha_{1}} \times Z_{1} \bar{\beta}\right) \cup\left(Y_{0}^{\alpha_{1}} \times \breve{D} \bar{\beta}\right) \\
& =\left(Y_{4}^{\alpha_{1}} \times Z_{4}\right) \cup\left(Y_{3}^{\alpha_{1}} \times \breve{D}\right) \cup\left(Y_{2}^{\alpha_{1}} \times \breve{D}\right) \cup\left(Y_{1}^{\alpha_{1}} \times Z_{1}\right) \cup\left(Y_{0}^{\alpha_{1}} \times \breve{D}\right) \\
& =\left(Y_{4}^{\alpha_{1}} \times Z_{4}\right) \cup\left(Y_{1}^{\alpha_{1}} \times Z_{1}\right) \cup\left(\left(Y_{3}^{\alpha_{1}} \cup Y_{2}^{\alpha_{1}} \cup Y_{0}^{\alpha_{1}}\right) \times \breve{D}\right) \\
& =\left(Y_{4}^{\alpha} \times Z_{4}\right) \cup\left(Y_{1}^{\alpha} \times Z_{1}\right) \cup\left(Y_{0}^{\alpha} \times \breve{D}\right) .
\end{aligned}
$$

2. Let $V\left(X^{*}, \alpha\right)=\left\{Z_{4}, Z_{3}, \breve{D}\right\}$. Then binary relation $\alpha$ has representation of the form 
$\alpha=\left(Y_{4}^{\alpha} \times Z_{4}\right) \cup\left(Y_{3}^{\alpha} \times Z_{3}\right) \cup\left(Y_{0}^{\alpha} \times \breve{D}\right)$. In this case suppose that

$$
\bar{\beta}_{1}=\left(\begin{array}{ccccc}
P_{0} & P_{1} & P_{2} & P_{3} & P_{4} \\
\varnothing & Z_{3} & Z_{4} & Z_{2} & \varnothing
\end{array}\right)
$$

and $\bar{\beta}_{2}$ are mapping of the set $X \backslash \breve{D}$ on the set $D \backslash\left\{Z_{4}, Z_{3}, Z_{2}\right\}$. Then

$$
\begin{aligned}
& \bar{\beta}=\left(P_{0} \times \varnothing\right) \cup\left(P_{1} \times Z_{3}\right) \cup\left(P_{2} \times Z_{4}\right) \cup\left(P_{3} \times Z_{2}\right) \cup\left(P_{4} \times \varnothing\right) \cup \bigcup_{t^{\prime} \in X \backslash \bar{D}}\left(\left\{t^{\prime}\right\} \times f\left(t^{\prime}\right)\right), \\
& \alpha_{1}=\left(Y_{4}^{\alpha_{1}} \times Z_{4}\right) \cup\left(Y_{3}^{\alpha_{1}} \times Z_{3}\right) \cup\left(Y_{2}^{\alpha_{1}} \times Z_{2}\right) \cup\left(Y_{1}^{\alpha_{1}} \times Z_{1}\right) \cup\left(Y_{0}^{\alpha_{1}} \times \breve{D}\right),
\end{aligned}
$$

where $Y_{4}^{\alpha_{1}}, Y_{3}^{\alpha_{1}}, Y_{2}^{\alpha_{1}}, Y_{1}^{\alpha_{1}} \notin\{\varnothing\}, Y_{4}^{\alpha_{1}}=Y_{4}^{\alpha}, Y_{3}^{\alpha_{1}}=Y_{3}^{\alpha}$ and $Y_{2}^{\alpha_{1}} \cup Y_{1}^{\alpha_{1}} \cup Y_{0}^{\alpha_{1}}=Y_{0}^{\alpha}$, then it is easy to see, that $\alpha_{1}, \bar{\beta} \in B$ since $V\left(D, \alpha_{1}\right)=V(D, \bar{\beta})=D$. From the formal equality and equalities (11) and (5) we have:

$$
\begin{aligned}
Z_{4} \bar{\beta} & =\left(P_{0} \cup P_{2}\right) \bar{\beta}=P_{0} \bar{\beta} \cup P_{2} \bar{\beta}=\varnothing \cup Z_{4}=Z_{4} ; \\
Z_{3} \bar{\beta} & =\left(P_{0} \cup P_{1} \cup P_{2} \cup P_{4}\right) \bar{\beta}=P_{0} \bar{\beta} \cup P_{1} \bar{\beta} \cup P_{2} \bar{\beta} \cup P_{4} \bar{\beta} \\
& =\varnothing \cup Z_{3} \cup Z_{4} \cup \varnothing=Z_{3} ; \\
Z_{2} \bar{\beta} & =\left(P_{0} \cup P_{1} \cup P_{3} \cup P_{4}\right) \bar{\beta}=P_{0} \bar{\beta} \cup P_{1} \bar{\beta} \cup P_{3} \bar{\beta} \cup P_{4} \bar{\beta} \\
& =\varnothing \cup Z_{3} \cup Z_{2} \cup \varnothing=\breve{D} ; \\
Z_{1} \bar{\beta} & =\left(P_{0} \cup P_{2} \cup P_{3} \cup P_{4}\right) \bar{\beta}=P_{0} \bar{\beta} \cup P_{2} \bar{\beta} \cup P_{3} \bar{\beta} \cup P_{4} \bar{\beta} \\
& =\varnothing \cup Z_{4} \cup Z_{2} \cup \varnothing=\breve{D} ; \\
\bar{D} \bar{\beta} & =\left(P_{0} \cup P_{1} \cup P_{2} \cup P_{3} \cup P_{4}\right) \bar{\beta}=P_{0} \bar{\beta} \cup P_{1} \bar{\beta} \cup P_{2} \bar{\beta} \cup P_{3} \bar{\beta} \cup P_{4} \bar{\beta} \\
& =\varnothing \cup Z_{3} \cup Z_{4} \cup Z_{2} \cup \varnothing=\breve{D} ; \\
\alpha & =\alpha_{1} \circ \bar{\beta} \\
& =\left(Y_{4}^{\alpha_{1}} \times Z_{4} \bar{\beta}\right) \cup\left(Y_{3}^{\alpha_{1}} \times Z_{3} \bar{\beta}\right) \cup\left(Y_{2}^{\alpha_{1}} \times Z_{2} \bar{\beta}\right) \cup\left(Y_{1}^{\alpha_{1}} \times Z_{1} \bar{\beta}\right) \cup\left(Y_{0}^{\alpha_{1}} \times \breve{D} \bar{\beta}\right) \\
& =\left(Y_{4}^{\alpha_{1}} \times Z_{4}\right) \cup\left(Y_{3}^{\alpha_{1}} \times Z_{3}\right) \cup\left(Y_{2}^{\alpha_{1}} \times \breve{D}\right) \cup\left(Y_{1}^{\alpha_{1}} \times \breve{D}\right) \cup\left(Y_{0}^{\alpha_{1}} \times \breve{D}\right) \\
& =\left(Y_{4}^{\alpha_{1}} \times Z_{4}\right) \cup\left(Y_{3}^{\alpha_{1}} \times Z_{3}\right) \cup\left(\left(Y_{2}^{\alpha_{1}} \cup Y_{1}^{\alpha_{1}} \cup Y_{0}^{\alpha_{1}}\right) \times \breve{D}\right) \\
& =\left(Y_{4}^{\alpha} \times Z_{4}\right) \cup\left(Y_{3}^{\alpha} \times Z_{3}\right) \cup\left(Y_{0}^{\alpha} \times \breve{D}\right) .
\end{aligned}
$$

3. Let $V\left(X^{*}, \alpha\right)=\left\{Z_{4}, Z_{2}, \breve{D}\right\}$. Then binary relation $\alpha$ has representation of the form $\alpha=\left(Y_{4}^{\alpha} \times Z_{4}\right) \cup\left(Y_{2}^{\alpha} \times Z_{2}\right) \cup\left(Y_{0}^{\alpha} \times \breve{D}\right)$. In this case suppose that

$$
\bar{\beta}_{1}=\left(\begin{array}{lllll}
P_{0} & P_{1} & P_{2} & P_{3} & P_{4} \\
\varnothing & Z_{2} & Z_{4} & Z_{2} & \varnothing
\end{array}\right)
$$

and $\bar{\beta}_{2}$ are mapping of the set $X \backslash \breve{D}$ on the set $D \backslash\left\{Z_{4}, Z_{2}\right\}$. Then

$$
\begin{aligned}
& \bar{\beta}=\left(P_{0} \times \varnothing\right) \cup\left(P_{1} \times Z_{2}\right) \cup\left(P_{2} \times Z_{4}\right) \cup\left(P_{3} \times Z_{2}\right) \cup\left(P_{4} \times \varnothing\right) \cup \bigcup_{t^{\prime} \in X \backslash \bar{D}}\left(\left\{t^{\prime}\right\} \times f\left(t^{\prime}\right)\right), \\
& \alpha_{1}=\left(Y_{4}^{\alpha_{1}} \times Z_{4}\right) \cup\left(Y_{3}^{\alpha_{1}} \times Z_{3}\right) \cup\left(Y_{2}^{\alpha_{1}} \times Z_{2}\right) \cup\left(Y_{1}^{\alpha_{1}} \times Z_{1}\right) \cup\left(Y_{0}^{\alpha_{1}} \times \breve{D}\right),
\end{aligned}
$$

where $Y_{4}^{\alpha_{1}}, Y_{3}^{\alpha_{1}}, Y_{2}^{\alpha_{1}}, Y_{1}^{\alpha_{1}} \notin\{\varnothing\}, Y_{4}^{\alpha_{1}}=Y_{4}^{\alpha}, Y_{2}^{\alpha_{1}}=Y_{2}^{\alpha}$ and $Y_{3}^{\alpha_{1}} \cup Y_{1}^{\alpha_{1}} \cup Y_{0}^{\alpha_{1}}=Y_{0}^{\alpha}$, then it is easy to see, that $\alpha_{1}, \bar{\beta} \in B$ since $V\left(D, \alpha_{1}\right)=V(D, \bar{\beta})=D$. From the formal equality and equalities (12) and (5) we have: 


$$
\begin{aligned}
& Z_{4} \bar{\beta}=\left(P_{0} \cup P_{2}\right) \bar{\beta}=P_{0} \bar{\beta} \cup P_{2} \bar{\beta}=\varnothing \cup Z_{4}=Z_{4} ; \\
& Z_{3} \bar{\beta}=\left(P_{0} \cup P_{1} \cup P_{2} \cup P_{4}\right) \bar{\beta}=P_{0} \bar{\beta} \cup P_{1} \bar{\beta} \cup P_{2} \bar{\beta} \cup P_{4} \bar{\beta}=\varnothing \cup Z_{2} \cup Z_{4} \cup \varnothing=\breve{D} ; \\
& Z_{2} \bar{\beta}=\left(P_{0} \cup P_{1} \cup P_{3} \cup P_{4}\right) \bar{\beta}=P_{0} \bar{\beta} \cup P_{1} \bar{\beta} \cup P_{3} \bar{\beta} \cup P_{4} \bar{\beta}=\varnothing \cup Z_{2} \cup Z_{2} \cup \varnothing=Z_{2} ; \\
& Z_{1} \bar{\beta}=\left(P_{0} \cup P_{2} \cup P_{3} \cup P_{4}\right) \bar{\beta}=P_{0} \bar{\beta} \cup P_{2} \bar{\beta} \cup P_{3} \bar{\beta} \cup P_{4} \bar{\beta}=\varnothing \cup Z_{4} \cup Z_{2} \cup \varnothing=\bar{D} ; \\
& \bar{D} \bar{\beta}=\left(P_{0} \cup P_{1} \cup P_{2} \cup P_{3} \cup P_{4}\right) \bar{\beta}=P_{0} \bar{\beta} \cup P_{1} \bar{\beta} \cup P_{2} \bar{\beta} \cup P_{3} \bar{\beta} \cup P_{4} \bar{\beta} \\
& \quad=\varnothing \cup Z_{2} \cup Z_{4} \cup Z_{2} \cup \varnothing=\breve{D} ; \\
& \alpha=\alpha_{1} \circ \bar{\beta} \\
& =\left(Y_{4}^{\alpha_{1}} \times Z_{4} \bar{\beta}\right) \cup\left(Y_{3}^{\alpha_{1}} \times Z_{3} \bar{\beta}\right) \cup\left(Y_{2}^{\alpha_{1}} \times Z_{2} \bar{\beta}\right) \cup\left(Y_{1}^{\alpha_{1}} \times Z_{1} \bar{\beta}\right) \cup\left(Y_{0}^{\alpha_{1}} \times \bar{D} \bar{\beta}\right) \\
& =\left(Y_{4}^{\alpha_{1}} \times Z_{4}\right) \cup\left(Y_{3}^{\alpha_{1}} \times \bar{D}\right) \cup\left(Y_{2}^{\alpha_{1}} \times Z_{2}\right) \cup\left(Y_{1}^{\alpha_{1}} \times \bar{D}\right) \cup\left(Y_{0}^{\alpha_{1}} \times \bar{D}\right) \\
& =\left(Y_{4}^{\alpha_{1}} \times Z_{4}\right) \cup\left(Y_{2}^{\alpha_{1}} \times Z_{2}\right) \cup\left(\left(Y_{3}^{\alpha_{1}} \cup Y_{1}^{\alpha_{1}} \cup Y_{0}^{\alpha_{1}}\right) \times \breve{D}\right) \\
& =\left(Y_{4}^{\alpha} \times Z_{4}\right) \cup\left(Y_{2}^{\alpha} \times Z_{2}\right) \cup\left(Y_{0}^{\alpha} \times \breve{D}\right) .
\end{aligned}
$$

4. Let $V\left(X^{*}, \alpha\right)=\left\{Z_{1}, Z_{2}, \breve{D}\right\}$. Then binary relation $\alpha$ has representation of the form $\alpha=\left(Y_{1}^{\alpha} \times Z_{1}\right) \cup\left(Y_{2}^{\alpha} \times Z_{2}\right) \cup\left(Y_{0}^{\alpha} \times \breve{D}\right)$. In this case suppose that

$$
\bar{\beta}_{1}=\left(\begin{array}{lllll}
P_{0} & P_{1} & P_{2} & P_{3} & P_{4} \\
\varnothing & Z_{1} & Z_{2} & Z_{1} & \varnothing
\end{array}\right)
$$

and $\bar{\beta}_{2}$ are mapping of the set $X \backslash \breve{D}$ on the set $D \backslash\left\{Z_{2}, Z_{1}\right\}$. Then

$$
\begin{aligned}
& \bar{\beta}=\left(P_{0} \times \varnothing\right) \cup\left(P_{1} \times Z_{1}\right) \cup\left(P_{2} \times Z_{2}\right) \cup\left(P_{3} \times Z_{1}\right) \cup\left(P_{4} \times \varnothing\right) \cup \bigcup_{t^{\prime} \in X \backslash \bar{D}}\left(\left\{t^{\prime}\right\} \times f\left(t^{\prime}\right)\right), \\
& \alpha_{1}=\left(Y_{4}^{\alpha_{1}} \times Z_{4}\right) \cup\left(Y_{3}^{\alpha_{1}} \times Z_{3}\right) \cup\left(Y_{2}^{\alpha_{1}} \times Z_{2}\right) \cup\left(Y_{1}^{\alpha_{1}} \times Z_{1}\right) \cup\left(Y_{0}^{\alpha_{1}} \times \breve{D}\right),
\end{aligned}
$$

where $Y_{4}^{\alpha_{1}}, Y_{3}^{\alpha_{1}}, Y_{2}^{\alpha_{1}}, Y_{1}^{\alpha_{1}} \notin\{\varnothing\}, Y_{2}^{\alpha_{1}}=Y_{1}^{\alpha}, Y_{4}^{\alpha_{1}}=Y_{2}^{\alpha}$ and $Y_{3}^{\alpha_{1}} \cup Y_{1}^{\alpha_{1}} \cup Y_{0}^{\alpha_{1}}=Y_{0}^{\alpha}$, then it is easy to see, that $\alpha_{1}, \bar{\beta} \in B$ since $V\left(D, \alpha_{1}\right)=V(D, \bar{\beta})=D$. From the formal equality and equalities (13) and (5) we have:

$$
\begin{aligned}
& Z_{4} \bar{\beta}=\left(P_{0} \cup P_{2}\right) \bar{\beta}=P_{0} \bar{\beta} \cup P_{2} \bar{\beta}=\varnothing \cup Z_{2}=Z_{2} ; \\
& Z_{3} \bar{\beta}=\left(P_{0} \cup P_{1} \cup P_{2} \cup P_{4}\right) \bar{\beta}=P_{0} \bar{\beta} \cup P_{1} \bar{\beta} \cup P_{2} \bar{\beta} \cup P_{4} \bar{\beta}=\varnothing \cup Z_{1} \cup Z_{2} \cup \varnothing=\breve{D} ; \\
& Z_{2} \bar{\beta}=\left(P_{0} \cup P_{1} \cup P_{3} \cup P_{4}\right) \bar{\beta}=P_{0} \bar{\beta} \cup P_{1} \bar{\beta} \cup P_{3} \bar{\beta} \cup P_{4} \bar{\beta}=\varnothing \cup Z_{1} \cup Z_{1} \cup \varnothing=Z_{1} ; \\
& Z_{1} \bar{\beta}=\left(P_{0} \cup P_{2} \cup P_{3} \cup P_{4}\right) \bar{\beta}=P_{0} \bar{\beta} \cup P_{2} \bar{\beta} \cup P_{3} \bar{\beta} \cup P_{4} \bar{\beta}=\varnothing \cup Z_{2} \cup Z_{1} \cup \varnothing=\bar{D} ; \\
& \bar{D} \bar{\beta}=\left(P_{0} \cup P_{1} \cup P_{2} \cup P_{3} \cup P_{4}\right) \bar{\beta}=P_{0} \bar{\beta} \cup P_{1} \bar{\beta} \cup P_{2} \bar{\beta} \cup P_{3} \bar{\beta} \cup P_{4} \bar{\beta} \\
& \quad=\varnothing \cup Z_{1} \cup Z_{2} \cup Z_{1} \cup \varnothing=\bar{D} ; \\
& \alpha=\alpha_{1} \circ \bar{\beta} \\
& =\left(Y_{4}^{\alpha_{1}} \times Z_{4} \bar{\beta}\right) \cup\left(Y_{3}^{\alpha_{1}} \times Z_{3} \bar{\beta}\right) \cup\left(Y_{2}^{\alpha_{1}} \times Z_{2} \bar{\beta}\right) \cup\left(Y_{1}^{\alpha_{1}} \times Z_{1} \bar{\beta}\right) \cup\left(Y_{0}^{\alpha_{1}} \times \breve{D} \bar{\beta}\right) \\
& =\left(Y_{4}^{\alpha_{1}} \times Z_{2}\right) \cup\left(Y_{3}^{\alpha_{1}} \times \breve{D}\right) \cup\left(Y_{2}^{\alpha_{1}} \times Z_{1}\right) \cup\left(Y_{1}^{\alpha_{1}} \times \breve{D}\right) \cup\left(Y_{0}^{\alpha_{1}} \times \breve{D}\right) \\
& =\left(Y_{2}^{\alpha_{1}} \times Z_{1}\right) \cup\left(Y_{4}^{\alpha_{1}} \times Z_{2}\right) \cup\left(\left(Y_{3}^{\alpha_{1}} \cup Y_{1}^{\alpha_{1}} \cup Y_{0}^{\alpha_{1}}\right) \times \breve{D}\right) \\
& =\left(Y_{1}^{\alpha} \times Z_{1}\right) \cup\left(Y_{2}^{\alpha} \times Z_{2}\right) \cup\left(Y_{0}^{\alpha} \times \breve{D}\right) .
\end{aligned}
$$

5. Let $V\left(X^{*}, \alpha\right)=\left\{Z_{1}, Z_{3}, \breve{D}\right\}$. Then binary relation $\alpha$ has representation of the form $\alpha=\left(Y_{1}^{\alpha} \times Z_{1}\right) \cup\left(Y_{3}^{\alpha} \times Z_{3}\right) \cup\left(Y_{0}^{\alpha} \times \breve{D}\right)$. In this case suppose that

$$
\bar{\beta}_{1}=\left(\begin{array}{lllll}
P_{0} & P_{1} & P_{2} & P_{3} & P_{4} \\
\varnothing & Z_{3} & Z_{1} & Z_{3} & \varnothing
\end{array}\right)
$$


and $\bar{\beta}_{2}$ are mapping of the set $X \backslash \breve{D}$ on the set $D \backslash\left\{Z_{3}, Z_{1}\right\}$. Then

$$
\begin{aligned}
& \bar{\beta}=\left(P_{0} \times \varnothing\right) \cup\left(P_{1} \times Z_{3}\right) \cup\left(P_{2} \times Z_{1}\right) \cup\left(P_{3} \times Z_{3}\right) \cup\left(P_{4} \times \varnothing\right) \cup \bigcup_{t^{\prime} \in X \backslash \bar{D}}\left(\left\{t^{\prime}\right\} \times f\left(t^{\prime}\right)\right), \\
& \alpha_{1}=\left(Y_{4}^{\alpha_{1}} \times Z_{4}\right) \cup\left(Y_{3}^{\alpha_{1}} \times Z_{3}\right) \cup\left(Y_{2}^{\alpha_{1}} \times Z_{2}\right) \cup\left(Y_{1}^{\alpha_{1}} \times Z_{1}\right) \cup\left(Y_{0}^{\alpha_{1}} \times \breve{D}\right),
\end{aligned}
$$

where $Y_{4}^{\alpha_{1}}, Y_{3}^{\alpha_{1}}, Y_{2}^{\alpha_{1}}, Y_{1}^{\alpha_{1}} \notin\{\varnothing\}, Y_{4}^{\alpha_{1}}=Y_{1}^{\alpha}, Y_{2}^{\alpha_{1}}=Y_{3}^{\alpha}$ and $Y_{3}^{\alpha_{1}} \cup Y_{1}^{\alpha_{1}} \cup Y_{0}^{\alpha_{1}}=Y_{0}^{\alpha}$, then it is easy to see, that $\alpha_{1}, \bar{\beta} \in B$ since $V\left(D, \alpha_{1}\right)=V(D, \bar{\beta})=D$. From the formal equality and equalities (14) and (5) we have:

$$
\begin{aligned}
Z_{4} \bar{\beta} & =\left(P_{0} \cup P_{2}\right) \bar{\beta}=P_{0} \bar{\beta} \cup P_{2} \bar{\beta}=\varnothing \cap Z_{1}=Z_{1} ; \\
Z_{3} \bar{\beta} & =\left(P_{0} \cup P_{1} \cup P_{2} \cup P_{4}\right) \bar{\beta}=P_{0} \bar{\beta} \cup P_{1} \bar{\beta} \cup P_{2} \bar{\beta} \cup P_{4} \bar{\beta}=\varnothing \cup Z_{3} \cup Z_{1} \cup \varnothing=\breve{D} ; \\
Z_{2} \bar{\beta} & =\left(P_{0} \cup P_{1} \cup P_{3} \cup P_{4}\right) \bar{\beta}=P_{0} \bar{\beta} \cup P_{1} \bar{\beta} \cup P_{3} \bar{\beta} \cup P_{4} \bar{\beta}=\varnothing \cup Z_{3} \cup Z_{3} \cup \varnothing=Z_{3} ; \\
Z_{1} \bar{\beta} & =\left(P_{0} \cup P_{2} \cup P_{3} \cup P_{4}\right) \bar{\beta}=P_{0} \bar{\beta} \cup P_{2} \bar{\beta} \cup P_{3} \bar{\beta} \cup P_{4} \bar{\beta}=\varnothing \cup Z_{1} \cup Z_{3} \cup \varnothing=\bar{D} ; \\
\bar{D} \bar{\beta} & =\left(P_{0} \cup P_{1} \cup P_{2} \cup P_{3} \cup P_{4}\right) \bar{\beta}=P_{0} \bar{\beta} \cup P_{1} \bar{\beta} \cup P_{2} \bar{\beta} \cup P_{3} \bar{\beta} \cup P_{4} \bar{\beta} \\
& =\varnothing \cup Z_{3} \cup Z_{1} \cup Z_{3} \cup \varnothing=\bar{D} ; \\
\alpha= & \alpha_{1} \circ \bar{\beta} \\
= & \left(Y_{4}^{\alpha_{1}} \times Z_{4} \bar{\beta}\right) \cup\left(Y_{3}^{\alpha_{1}} \times Z_{3} \bar{\beta}\right) \cup\left(Y_{2}^{\alpha_{1}} \times Z_{2} \bar{\beta}\right) \cup\left(Y_{1}^{\alpha_{1}} \times Z_{1} \bar{\beta}\right) \cup\left(Y_{0}^{\alpha_{1}} \times \breve{D} \bar{\beta}\right) \\
= & \left(Y_{4}^{\alpha_{1}} \times Z_{1}\right) \cup\left(Y_{3}^{\alpha_{1}} \times \breve{D}\right) \cup\left(Y_{2}^{\alpha_{1}} \times Z_{3}\right) \cup\left(Y_{1}^{\alpha_{1}} \times \breve{D}\right) \cup\left(Y_{0}^{\alpha_{1}} \times \breve{D}\right) \\
= & \left(Y_{4}^{\alpha_{1}} \times Z_{1}\right) \cup\left(Y_{2}^{\alpha_{1}} \times Z_{3}\right) \cup\left(\left(Y_{3}^{\alpha_{1}} \cup Y_{1}^{\alpha_{1}} \cup Y_{0}^{\alpha_{1}}\right) \times \breve{D}\right) \\
= & \left(Y_{1}^{\alpha} \times Z_{1}\right) \cup\left(Y_{3}^{\alpha} \times Z_{3}\right) \cup\left(Y_{0}^{\alpha} \times \breve{D}\right) .
\end{aligned}
$$

6. Let $V\left(X^{*}, \alpha\right)=\left\{Z_{2}, Z_{3}, \breve{D}\right\}$. Then binary relation $\alpha$ has representation of the form $\alpha=\left(Y_{2}^{\alpha} \times Z_{2}\right) \cup\left(Y_{3}^{\alpha} \times Z_{3}\right) \cup\left(Y_{0}^{\alpha} \times \breve{D}\right)$. In this case suppose that

$$
\bar{\beta}_{1}=\left(\begin{array}{lllll}
P_{0} & P_{1} & P_{2} & P_{3} & P_{4} \\
\varnothing & Z_{3} & Z_{2} & Z_{3} & \varnothing
\end{array}\right)
$$

and $\bar{\beta}_{2}$ are mapping of the set $X \backslash \breve{D}$ on the set $D \backslash\left\{Z_{3}, Z_{2}\right\}$. Then

$$
\begin{aligned}
& \bar{\beta}=\left(P_{0} \times \varnothing\right) \cup\left(P_{1} \times Z_{3}\right) \cup\left(P_{2} \times Z_{2}\right) \cup\left(P_{3} \times Z_{3}\right) \cup\left(P_{4} \times \varnothing\right) \cup \bigcup_{t^{\prime} \in X \backslash \bar{D}}\left(\left\{t^{\prime}\right\} \times f\left(t^{\prime}\right)\right) \\
& \alpha_{1}=\left(Y_{4}^{\alpha_{1}} \times Z_{4}\right) \cup\left(Y_{3}^{\alpha_{1}} \times Z_{3}\right) \cup\left(Y_{2}^{\alpha_{1}} \times Z_{2}\right) \cup\left(Y_{1}^{\alpha_{1}} \times Z_{1}\right) \cup\left(Y_{0}^{\alpha_{1}} \times \bar{D}\right),
\end{aligned}
$$

where $Y_{4}^{\alpha_{1}}, Y_{3}^{\alpha_{1}}, Y_{2}^{\alpha_{1}}, Y_{1}^{\alpha_{1}} \notin\{\varnothing\}, Y_{4}^{\alpha_{1}}=Y_{2}^{\alpha}, Y_{2}^{\alpha_{1}}=Y_{3}^{\alpha}$ and $Y_{3}^{\alpha_{1}} \cup Y_{1}^{\alpha_{1}} \cup Y_{0}^{\alpha_{1}}=Y_{0}^{\alpha}$, then it is easy to see, that $\alpha_{1}, \bar{\beta} \in B$ since $V\left(D, \alpha_{1}\right)=V(D, \bar{\beta})=D$. From the formal equality and equalities (15) and (5) we have:

$$
\begin{aligned}
& Z_{4} \bar{\beta}=\left(P_{0} \cup P_{2}\right) \bar{\beta}=P_{0} \bar{\beta} \cup P_{2} \bar{\beta}=\varnothing \cup Z_{2}=Z_{2} ; \\
& Z_{3} \bar{\beta}=\left(P_{0} \cup P_{1} \cup P_{2} \cup P_{4}\right) \bar{\beta}=P_{0} \bar{\beta} \cup P_{1} \bar{\beta} \cup P_{2} \bar{\beta} \cup P_{4} \bar{\beta}=\varnothing \cup Z_{3} \cup Z_{2} \cup \varnothing=\breve{D} ; \\
& Z_{2} \bar{\beta}=\left(P_{0} \cup P_{1} \cup P_{3} \cup P_{4}\right) \bar{\beta}=P_{0} \bar{\beta} \cup P_{1} \bar{\beta} \cup P_{3} \bar{\beta} \cup P_{4} \bar{\beta}=\varnothing \cup Z_{3} \cup Z_{3} \cup \varnothing=Z_{3} ; \\
& Z_{1} \bar{\beta}=\left(P_{0} \cup P_{2} \cup P_{3} \cup P_{4}\right) \bar{\beta}=P_{0} \bar{\beta} \cup P_{2} \bar{\beta} \cup P_{3} \bar{\beta} \cup P_{4} \bar{\beta}=\varnothing \cup Z_{2} \cup Z_{3} \cup \varnothing=\bar{D} ; \\
& \bar{D} \bar{\beta}=\left(P_{0} \cup P_{1} \cup P_{2} \cup P_{3} \cup P_{4}\right) \bar{\beta}=P_{0} \bar{\beta} \cup P_{1} \bar{\beta} \cup P_{2} \bar{\beta} \cup P_{3} \bar{\beta} \cup P_{4} \bar{\beta} \\
& \quad=\varnothing \cup Z_{3} \cup Z_{2} \cup Z_{3} \cup \varnothing=\bar{D} ; \\
& \alpha=\alpha_{1} \circ \bar{\beta} \\
& =\left(Y_{4}^{\alpha_{1}} \times Z_{4} \bar{\beta}\right) \cup\left(Y_{3}^{\alpha_{1}} \times Z_{3} \bar{\beta}\right) \cup\left(Y_{2}^{\alpha_{1}} \times Z_{2} \bar{\beta}\right) \cup\left(Y_{1}^{\alpha_{1}} \times Z_{1} \bar{\beta}\right) \cup\left(Y_{0}^{\alpha_{1}} \times \breve{D} \bar{\beta}\right) \\
& =\left(Y_{4}^{\alpha_{1}} \times Z_{2}\right) \cup\left(Y_{3}^{\alpha_{1}} \times \breve{D}\right) \cup\left(Y_{2}^{\alpha_{1}} \times Z_{3}\right) \cup\left(Y_{1}^{\alpha_{1}} \times \breve{D}\right) \cup\left(Y_{0}^{\alpha_{1}} \times \breve{D}\right) \\
& =\left(Y_{4}^{\alpha_{1}} \times Z_{2}\right) \cup\left(Y_{2}^{\alpha_{1}} \times Z_{3}\right) \cup\left(\left(Y_{3}^{\alpha_{1}} \cup Y_{1}^{\alpha_{1}} \cup Y_{0}^{\alpha_{1}}\right) \times \bar{D}\right) \\
& =\left(Y_{2}^{\alpha} \times Z_{2}\right) \cup\left(Y_{3}^{\alpha} \times Z_{3}\right) \cup\left(Y_{0}^{\alpha} \times \breve{D}\right) .
\end{aligned}
$$


7. Let $V\left(X^{*}, \alpha\right)=\left\{Z_{4}, Z_{3}, Z_{1}, \breve{D}\right\}$. Then binary relation $\alpha$ has representation of the form $\alpha=\left(Y_{4}^{\alpha} \times Z_{4}\right) \cup\left(Y_{3}^{\alpha} \times Z_{3}\right) \cup\left(Y_{1}^{\alpha} \times Z_{1}\right) \cup\left(Y_{0}^{\alpha} \times \breve{D}\right)$. In this case suppose that

$$
\bar{\beta}_{1}=\left(\begin{array}{lllll}
P_{0} & P_{1} & P_{2} & P_{3} & P_{4} \\
\varnothing & Z_{3} & Z_{4} & Z_{1} & \varnothing
\end{array}\right)
$$

and $\bar{\beta}_{2}$ are mapping of the set $X \backslash \breve{D}$ on the set $D \backslash\left\{Z_{4}, Z_{3}, Z_{1}\right\}$. Then

$$
\begin{aligned}
& \bar{\beta}=\left(P_{0} \times \varnothing\right) \cup\left(P_{1} \times Z_{3}\right) \cup\left(P_{2} \times Z_{4}\right) \cup\left(P_{3} \times Z_{1}\right) \cup\left(P_{4} \times \varnothing\right) \cup \bigcup_{t^{\prime} \in X \backslash D}\left(\left\{t^{\prime}\right\} \times f\left(t^{\prime}\right)\right), \\
& \alpha_{1}=\left(Y_{4}^{\alpha_{1}} \times Z_{4}\right) \cup\left(Y_{3}^{\alpha_{1}} \times Z_{3}\right) \cup\left(Y_{2}^{\alpha_{1}} \times Z_{2}\right) \cup\left(Y_{1}^{\alpha_{1}} \times Z_{1}\right) \cup\left(Y_{0}^{\alpha_{1}} \times \breve{D}\right),
\end{aligned}
$$

where $Y_{4}^{\alpha_{1}}, Y_{3}^{\alpha_{1}}, Y_{2}^{\alpha_{1}}, Y_{1}^{\alpha_{1}} \notin\{\varnothing\}, Y_{4}^{\alpha_{1}}=Y_{4}^{\alpha}, Y_{3}^{\alpha_{1}}=Y_{3}^{\alpha}, Y_{1}^{\alpha_{1}}=Y_{1}^{\alpha}$ and $Y_{2}^{\alpha_{1}} \cup Y_{0}^{\alpha_{1}}=Y_{0}^{\alpha}$, then it is easy to see, that $\alpha_{1}, \bar{\beta} \in B$ since $V\left(D, \alpha_{1}\right)=V(D, \bar{\beta})=D$. From the formal equality and equalities (16) and (5) we have:

$$
\begin{aligned}
& Z_{4} \bar{\beta}=\left(P_{0} \cup P_{2}\right) \bar{\beta}=P_{0} \bar{\beta} \cup P_{2} \bar{\beta}=\varnothing \cup Z_{4}=Z_{4} ; \\
& Z_{3} \bar{\beta}=\left(P_{0} \cup P_{1} \cup P_{2} \cup P_{4}\right) \bar{\beta}=P_{0} \bar{\beta} \cup P_{1} \bar{\beta} \cup P_{2} \bar{\beta} \cup P_{4} \bar{\beta}=\varnothing \cup Z_{3} \cup Z_{4} \cup \varnothing=Z_{3} ; \\
& Z_{2} \bar{\beta}=\left(P_{0} \cup P_{1} \cup P_{3} \cup P_{4}\right) \bar{\beta}=P_{0} \bar{\beta} \cup P_{1} \bar{\beta} \cup P_{3} \bar{\beta} \cup P_{4} \bar{\beta}=\varnothing \cup Z_{3} \cup Z_{1} \cup \varnothing=\bar{D} ; \\
& Z_{1} \bar{\beta}=\left(P_{0} \cup P_{2} \cup P_{3} \cup P_{4}\right) \bar{\beta}=P_{0} \bar{\beta} \cup P_{2} \bar{\beta} \cup P_{3} \bar{\beta} \cup P_{4} \bar{\beta}=\varnothing \cup Z_{4} \cup Z_{1} \cup \varnothing=Z_{1} ; \\
& \bar{D} \bar{\beta}=\left(P_{0} \cup P_{1} \cup P_{2} \cup P_{3} \cup P_{4}\right) \bar{\beta}=P_{0} \bar{\beta} \cup P_{1} \bar{\beta} \cup P_{2} \bar{\beta} \cup P_{3} \bar{\beta} \cup P_{4} \bar{\beta} \\
& \quad=\varnothing \cup Z_{3} \cup Z_{4} \cup Z_{1} \cup \varnothing=\bar{D} ; \\
& \alpha=\alpha_{1} \circ \bar{\beta} \\
& =\left(Y_{4}^{\alpha_{1}} \times Z_{4} \bar{\beta}\right) \cup\left(Y_{3}^{\alpha_{1}} \times Z_{3} \bar{\beta}\right) \cup\left(Y_{2}^{\alpha_{1}} \times Z_{2} \bar{\beta}\right) \cup\left(Y_{1}^{\alpha_{1}} \times Z_{1} \bar{\beta}\right) \cup\left(Y_{0}^{\alpha_{1}} \times \breve{D} \bar{\beta}\right) \\
& =\left(Y_{4}^{\alpha_{1}} \times Z_{4}\right) \cup\left(Y_{3}^{\alpha_{1}} \times Z_{3}\right) \cup\left(Y_{2}^{\alpha_{1}} \times \breve{D}\right) \cup\left(Y_{1}^{\alpha_{1}} \times Z_{1}\right) \cup\left(Y_{0}^{\alpha_{1}} \times \breve{D}\right) \\
& =\left(Y_{4}^{\alpha_{1}} \times Z_{4}\right) \cup\left(Y_{3}^{\alpha_{1}} \times Z_{3}\right) \cup\left(Y_{1}^{\alpha_{1}} \times Z_{1}\right) \cup\left(\left(Y_{2}^{\alpha_{1}} \cup Y_{0}^{\alpha_{1}}\right) \times \breve{D}\right) \\
& =\left(Y_{4}^{\alpha} \times Z_{4}\right) \cup\left(Y_{3}^{\alpha} \times Z_{3}\right) \cup\left(Y_{1}^{\alpha} \times Z_{1}\right) \cup\left(Y_{0}^{\alpha} \times \breve{D}\right) .
\end{aligned}
$$

\section{References}

[1] Diasamidze, Ya. and Makharadze, Sh. (2013) Complete Semigroups of Binary Relations. Cityplace Kriter, CountryRegion Turkey, 520 p.

[2] Davedze, M.Kh. (1968) Generating Sets of Some Subsemigroups of the Semigroup of All Binary Relations in a Finite Set. Proc. A. I. Hertzen Leningrad State Polytechn. Inst., 387, 92-100. (In Russian)

[3] Davedze, M.Kh. (1971) A Semigroup Generated by the Set of All Binary Relations in a Finite Set. XIth All-Union Algebraic Colloquium, Abstracts of Reports, Kishinev, 193-194. (In Russian)

[4] Davedze, M.Kh. (1968) Generating Sets of the Subsemigroup of All Binary Relations in a Finite Set. Doklady AN BSSR, 12, 765-768. (In Russian)

[5] Givradze, O. (2010) Irreducible Generating Sets of Complete Semigroups of Unions $B_{X}(D)$ Defined by Semilattices of Class $\sum_{2}(X, 4)$. Proceedings of the International Conference “Modern Algebra and Its Aplications”, Batumi.

[6] Givradze, O. (2011) Irreducible Generating Sets of Complete Semigroups of Unions $B_{X}(D)$ Defined by Semilattices of Class in Case, When $X=\breve{D}$ and $\left|\breve{D} \backslash Z_{3}\right|>1$. Proceedings of the International Conference "Modern Algebra and Its Aplications", Batumi. 